\title{
COINCIDENCE THEOREMS FOR NONLINEAR HYBRID CONTRACTIONS
}

\section{Y.J. CHO}

Deptartment of Mathematics Gyeongsang National University Chinju 660-701, Korea

\section{B. FISHER}

Department of Mathematics and Computer Science

University of Leicester

Leicester LE1 7RH, England

\section{G.S. GENGA}

Deptartment of Mathematics Sunchon National University Sunchon 540-742, Korea

(Received June 5, 1995 and in revised form December 12, 1995)

ABSTRACT. In this paper, we give some common fixed point theorems for single-valued mappings and multi-valued mappings satisfying a rational inequality Our theorems generalize some results of B Fisher, M L. Diviccaro et al. and V Popa

KEY WORDS AND PHRASES: Compatible mappings, weakly commuting mappings, coincidence points and fixed points.

1991 AMS SUBJECT CLASSIFICATION CODES: 54H25, 47H10.

\section{INTRODUCTION}

Let $(X, d)$ be a metric space and let $f$ and $g$ be mappings from $X$ into itself. In [1], S Sessa defined $f$ and $g$ to be weakly commuting if

$$
d(g f x, f g x) \leq d(g x, f x)
$$

for all $x$ in $X$ It can be seen that two commuting mappings are weakly commuting, but the converse is false as shown in the Example of [2]

Recently, G. Jungck [3] extended the concept of weak commutativity in the following way

DEFINITION 1.1. Let $f$ and $g$ be mappings from a metric space $(X, d)$ into itself. The mappings $f$ and $g$ are said to be compatible if

$$
\lim _{n \rightarrow \infty}\left(f g x_{n}, g f x_{n}\right)=0
$$

whenever $\left\{x_{n}\right\}$ is a sequence in $X$ such that $\lim _{n \rightarrow \infty} f x_{n}=\lim _{n \rightarrow \infty} g x_{n}=z$ for some $z$ in $X$

It is obvious that two weakly commuting mappings are compatible, but the converse is not true Some examples for this fact can be found in [3].

Recently, H. Kaneko [4] and S. L. Singh et al. [5] extended the concepts of weak commutativity and compatibility [6] for single-valued mappings to the setting of single-valued and multi-valued mappings, respectively

Let $(X, d)$ be a metric space and let $C B(X)$ denote the family of all nonempty closed and bounded subsets of $X$. Let $H$ be the Hausdorff metric on $C B(X)$ induced by the metric $d$, i e,

$$
H(A, B)=\max \left\{\sup _{x \in A} d(x, B), \sup _{y \in B} d(y, A)\right\}
$$


for $A, B \in C B(X)$, where $d(x, A)=\inf _{y \in A} d(x, y)$.

It is well-known that $(C B(X), H)$ is a metric space, and if a metric space $(X, d)$ is complete, then $(C H(X), H)$ is also complete.

Let $\delta(A, B)=\sup \{d(x, y): x \in A$ and $y \in B\}$ for all $A, B \in C B(X)$. If $A$ consists of a single point $a$, then we write $\delta(A, B)=\delta(a, B)$ If $\delta(A, B)=0$, then $A=B=\{a\}$ [7]

LEMMA 1.1 [8]. Let $A, B \in C B(X)$ and $k>1$. Then for each $a \in A$, there exists a point $b \in B$ such that $d(a, b) \leq k H(A, B)$.

Let $(X, d)$ be a metric space and let $f: X \rightarrow X$ and $S: X \rightarrow C B(X)$ be single-valued and multivalued mappings, respectively.

DEFINITION 1.2. The mappings $f$ and $S$ are said to be weakly commuting if for all $x \in X$, $f S x \in C B(X)$ and

$$
H(S f x, f S x) \leq d(f x, S x),
$$

where $H$ is the Hausdorff metric defined on $C B(X)$

DEFINITION 1.3. The mappings $f$ and $S$ are said to be compatible if

$$
\lim _{n \rightarrow \infty} d\left(f y_{n}, S f x_{n}\right)=0
$$

whenever $\left\{x_{n}\right\}$ and $\left\{y_{n}\right\}$ are sequences in $X$ such that $\lim _{n \rightarrow \infty} f x_{n}=\lim _{n \rightarrow \infty} y_{n}=z$ for some $z \in X$, where $y_{n} \in S x_{n}$ for $n=1,2, \ldots$.

REMARK 1.1. (1) Definition 13 is slightly different from the Kaneko's definition [6]

(2) If $S$ is a single-valued mapping on $X$ in Definitions 1.2 and 1.3, then Definitions 12 and 1.3 become the definitions of weak commutativity and compatibility for single-valued mappings

(3) If the mappings $f$ and $S$ are weakly commuting, then they are compatible, but the converse is not true

In fact, suppose that $f$ and $S$ are weakly commuting and let $\left\{x_{n}\right\}$ and $\left\{y_{n}\right\}$ be two sequences in $X$ such that $y_{n} \in S x_{n}$ for $n=1,2, \cdots$ and $\lim _{n \rightarrow \infty} f x_{n}=\lim _{n \rightarrow \infty} y_{n}=z$ for some $z \in X$ From $d\left(f x_{n}, S x_{n}\right) \leq d\left(f x_{n}, y_{n}\right)$, it follows that $\lim _{n \rightarrow \infty} d\left(f x_{n}, S x_{n}\right)=0$. Thus, since $f$ and $S$ are weakly commuting, we have

$$
\lim _{n \rightarrow \infty} H\left(S f x_{n}, f S x_{n}\right)=0 .
$$

On the other hand, since $d\left(f y_{n}, S f x_{n}\right) \leq H\left(f S x_{n}, S f x_{n}\right)$, we have

$$
\lim _{n \rightarrow \infty} d\left(f y_{n}, S f x_{n}\right)=0
$$

which means that $f$ and $S$ are compatible

EXAMPLE 1.1. Let $X=[1, \infty)$ be a set with the Euclidean metric $d$ and define $f x=2 x^{4}-1$ and $S x=\left[1, x^{2}\right]$ for all $x \geq 1$ Note that $f$ and $S$ are continuous and $S(X)=f(X)=X$ Let $\left\{x_{n}\right\}$ and $\left\{y_{n}\right\}$ be sequences in $X$ defined by $x_{n}=y_{n}=1$ for $n=1,2, \cdots$ Thus we have

$$
\lim _{n \rightarrow \infty} f x_{n}=\lim _{n \rightarrow \infty} y_{n}=1 \in X, y_{n} \in S x_{n} .
$$

On the other hand, we can show that $H\left(f S x_{n}, S f x_{n}\right)=2\left(x_{n}^{4}-1\right)^{2} \rightarrow 0$ if and only if $x_{n} \rightarrow 1$ as $n \rightarrow \infty$ and so, since $d\left(f y_{n}, S f x_{n}\right) \leq H\left(f S x_{n}, S f x_{n}\right)$, we have

$$
\lim _{n \rightarrow \infty} d\left(f y_{n}, S f x_{n}\right)=0 \text {. }
$$

Therefore, $f$ and $T$ are compatible, but $f$ and $T$ are not weakly commuting at $x=2$ 
We need the following lemmas for our main theorems, which is due to $G$ Jungck [2]

LEMMA 1.2. Let $f$ and $g$ be mappings from a metric space $(X, d)$ into itself If $f$ and $g$ are compatible and $f z=g z$ for some $z \in X$, then

$$
f g z=g g z=g f z=f f z .
$$

LEMMA 1.3. Let $f$ and $g$ be mappings from a metric space $(X, d)$ into itself If $f$ and $g$ are compatible and $f x_{n}, g x_{n} \rightarrow z$ for some $z \in X$, then we have the following

(1) $\lim _{n \rightarrow \infty} g f x_{n}=f z$ if $f$ is continuous at $z$,

(2) $f g z=g f z$ and $f z=g z$ if $f$ and $g$ are continuous at $z$

\section{COINCIDENCE THEOREMS FOR NONLINEAR HYBRID CONTRACTIONS}

In this section, we give some coincidence point theorems for nonlinear hybrid contractions, i e., contractive conditions involving single-valued and multi-valued mappings In the following Theorem 21 , $S(X)$ and $T(X)$ mean $S(X)=\cup_{x \in X} S x$ and $T(X)=\cup_{x \in X} T x$, respectively

THEOREM 2.1. Let $(X, d)$ be a complete metric space. Let $f, g: X \rightarrow X$ be continuous mappings and $S, T: X \rightarrow C B(X)$ be $H$-continuous multi-valued mappings such that

$T(X) \subset f(X)$ and $S(X) \subset g(X)$,

the pairs $f, S$ and $g, T$ are compatible mappings,

$$
H_{p}(S x, T y) \leq \frac{c d(f x, S x) d^{p}(g y, T y)+b d(f x, T y) d^{p}(g y, S x)}{\delta(f x, S x)+\delta(g y, T y)}
$$

for all $x, y \in X$ for which $\delta(f x, S x)+\delta(g y, T y) \neq 0$, where $p \geq 1, b \geq 0$ and $1<c<2$ Then there exists a point $z \in X$ such that $f x \in S z$ and $g z \in T z$, i.e, $z$ is a coincidence point of $f, S$ and of $g, T$

PROOF. Choose a real number $k$ such that $1<k<\left(\frac{2}{c}\right)^{\frac{1}{p}}$ and let $x_{0}$ be an arbitrary point in $X$ Since $S x_{0} \subset g(X)$, there exists a point $x_{1} \in X$ such that $g x_{1} \in S x_{0}$ and so there exists a point $y \in T x_{1}$ such that

$$
d\left(g x_{1}, y\right) \leq k H\left(S x_{0}, T x_{1}\right),
$$

which is possibly by Lemma 11 Since $T x_{1} \subset f(X)$, there exists a point $x_{2} \in X$ such that $y=f x_{2}$ and so we have

$$
d\left(g x_{1}, f x_{2}\right) \leq k H\left(S x_{0}, T x_{1}\right) .
$$

Similarly, there exists a point $x_{3} \in X$ such that $g x_{3} \in S x_{2}$ and

$$
d\left(g x_{3}, f x_{2}\right) \leq k H\left(S x_{2}, T x_{1}\right) .
$$

Inductively, we can obtain a sequence $\left\{x_{n}\right\}$ in $X$ such that

$$
\begin{gathered}
f x_{2 n} \in T x_{2 n-1}, \quad n \in N, \\
g x_{2 n+1} \in S x_{2 n}, \quad n \in N_{0}=N \cup\{0\}, \\
d\left(g x_{2 n+1}, f x_{2 n}\right) \leq k H\left(S x_{2 n}, T x_{2 n-1}\right), \quad n \in N, \\
d\left(g x_{2 n+1}, f x_{2 n+2}\right) \leq k H\left(S x_{2 n}, T x_{2 n+1}\right), \quad n \in N_{0},
\end{gathered}
$$

where $N$ denotes the set of positive integers.

First, suppose that for some $n \in N$

$$
\delta\left(f x_{2 n}, S x_{2 n}\right)+\delta\left(g x_{2 n+1}, T x_{2 n+1}\right)=0 .
$$


Then $f x_{2 n} \in S x_{2 n}$ and $g x_{2 n+1} \in T x_{2 n+1}$ and so $x_{2 n}$ is a coincidence point of $f$ and $S$ and $x_{2 n+1}$ is a coincidence point of $g$ and $T$.

Similarly, $\delta\left(f x_{2 n+2}, S x_{2 n+2}\right)+\delta\left(g x_{2 n+1}, T x_{2 n+1}\right)=0$ for some $n \in N$ implies that $x_{2 n+1}$ is a coincidence point of $g$ and $T$ and $x_{2 n+2}$ is a coincidence point of $f$ and $S$.

Now, suppose that $\delta\left(f x_{2 n}, S x_{2 n}\right)+\delta\left(g x_{2 n+1}, T x_{2 n+1}\right) \neq 0$ for $n \in N_{0}$ Then, by (23), we have

$$
\begin{aligned}
d^{p}\left(g x_{2 n+1}, f x_{2 n+2}\right) \\
\quad \leq k^{p} H^{p}\left(S x_{2 n}, T x_{2 n+1}\right) \\
\quad \leq k^{p} \frac{c d\left(f x_{2 n}, S x_{2 n}\right) d^{p}\left(g x_{2 n+1}, T x_{2 n+1}\right)+b d\left(f x_{2 n}, T x_{2 n+1}\right) d^{p}\left(g x_{2 n+1}, S x_{2 n}\right)}{\delta\left(f x_{2 n}, S x_{2 n}\right)+\delta\left(g x_{2 n+1}, T x_{2 n+1}\right)} \\
\quad \leq k^{p} \frac{c d\left(f x_{2 n}, g x_{2 n+1}\right) d^{p}\left(g x_{2 n+1}, f x_{2 n+2}\right)+b d\left(f x_{2 n}, f x_{2 n+2}\right) d^{p}\left(g x_{2 n+1}, g x_{2 n+1}\right)}{\delta\left(f x_{2 n}, S x_{2 n}\right)+\delta\left(g x_{2 n+1}, T x_{2 n+1}\right)} \\
\quad \leq k^{p} \frac{c d\left(f x_{2 n}, g x_{2 n+1}\right) d^{p}\left(g x_{2 n+1}, f x_{2 n+2}\right)}{d\left(f x_{2 n}, g x_{2 n+1}\right)+d\left(g x_{2 n+1}, f x_{2 n+2}\right)} .
\end{aligned}
$$

If $d\left(g x_{2 n+1}, f x_{2 n+2}\right)=0$ and $d\left(f x_{2 n}, g x_{2 n+1}\right) \neq 0$ in (24), then $g x_{2 n+1}=f x_{2 n+2} \in T x_{2 n+1}$ and so $x_{2 n+1}$ is a coincidence point of $g$ and $T$. But the case of $d\left(f x_{2 n}, g x_{2 n+1}\right)=0$ and $d\left(g x_{2 n+1}, f x_{2 n+2}\right) \neq 0$ in (2.4) cannot occur.

In fact, if $d\left(f x_{2 n}, g x_{2 n+1}\right)=0$ and $d\left(g x_{2 n+1}, f x_{2 n+2}\right) \neq 0$ in (2.4), then we have $d\left(g x_{2 n+1}, f x_{2 n+2}\right)=0$, which is impossible From (2.4), we have

$$
\begin{aligned}
d^{p}\left(g x_{2 n+1}, f x_{2 n+2}\right)[ & {\left[d\left(f x_{2 n}, g x_{2 n+1}\right)+d\left(g x_{2 n+1}, f x_{2 n+2}\right)\right] } \\
& \leq k^{p} c d\left(f x_{2 n}, g x_{2 n+1}\right) d^{p}\left(g x_{2 n+1}, f x_{2 n+2}\right),
\end{aligned}
$$

which implies that

$$
d\left(g x_{2 n+1}, f x_{2 n+1}\right) \leq\left(k^{p} c-1\right) d\left(f x_{2 n}, g x_{2 n+1}\right) .
$$

On the other hand, from (2.3), we have

$$
\begin{aligned}
d^{p}\left(g x_{2 n+3}, f x_{2 n+2}\right) \\
\quad \leq k^{p} H^{p}\left(S x_{2 n+2}, T x_{2 n+1}\right) \\
\quad \leq k^{p} \frac{c d\left(f x_{2 n+2}, S x_{2 n+2}\right) d^{p}\left(g x_{2 n+1}, T x_{2 n+1}\right)+b d\left(f x_{2 n+2}, T x_{2 n+1}\right) d^{p}\left(g x_{2 n+1}, S x_{2 n+2}\right)}{\delta\left(f x_{2 n+2}, S x_{2 n+2}\right)+\delta\left(g x_{2 n+1}, T x_{2 n+1}\right)} \\
\quad \leq k^{p} \frac{c d\left(f x_{2 n+2}, g x_{2 n+3}\right) d^{p}\left(g x_{2 n+1}, f x_{2 n+2}\right)}{d\left(f x_{2 n+2}, g x_{2 n+3}\right)+d\left(g x_{2 n+1}, f x_{2 n+2}\right)},
\end{aligned}
$$

which implies that, if $\alpha=d\left(x_{2 n+3}, f x_{2 n+2}\right) / d\left(f x_{2 n+2}, g x_{2 n+1}\right)$, then $\alpha^{p}+\alpha^{p-1} \leq k^{p} c$ Thus $\alpha<1$ and we have

$$
d\left(g x_{2 n+3}, f x_{2 n+2}\right) \leq d\left(f x_{2 n+2}, g x_{2 n+1}\right) .
$$

Repeating the above argument, since $0 \leq k^{p} c-1<1$, it follows that $\left\{g x_{1}, f x_{2}, g x_{3}, f x_{4}, \cdots\right.$, $\left.g x_{2 n-1}, g x_{2 n}, g x_{2 n+1}, \cdots\right\}$ is a Cauchy sequence in $X$. Since $(X, d)$ is a complete metric space, let $\lim _{n \rightarrow \infty}$ $g x_{2 n+1}=\lim _{n \rightarrow \infty} f x_{2 n}=z$.

Now, we will prove that $f z \in S z$, that is, $z$ is a coincidence point of $f$ and $S$. For every $n \in N$, we have

$$
d\left(f g x_{2 n+1}, S z\right) \leq d\left(f g x_{2 n+1}, S f x_{2 n}\right)+H\left(S f x_{2 n}, S z\right) .
$$

It follows from the $H$-continuity of $S$ that

$$
\lim _{n \rightarrow \infty} H\left(S f x_{2 n}, S z\right)=0
$$

since $f x_{2 n} \rightarrow z$ as $n \rightarrow \infty$. Since $f$ and $S$ are compatible mappings and $\lim _{n \rightarrow \infty} f z_{n}=\lim _{n \rightarrow \infty} y_{n}=z$, where $y_{n}=g x_{2 n+1} \in S x_{2 n}$ and $z_{n}=x_{2 n}$, we have 


$$
\lim _{n \rightarrow \infty} d\left(f y_{n}, S f z_{n}\right)=\lim _{n \rightarrow \infty} d\left(f g x_{2 n+1}, S f x_{2 n}\right)=0 .
$$

Thus, from (2 5), (2.6) and (2.7), we have $\lim _{n \rightarrow \infty} d\left(f g x_{2 n+1}, S z\right)=0$ and so, from

$$
d(f z, S z) \leq d\left(f z, f g x_{2 n+1}\right)+d\left(f g x_{2 n+1}, S z\right)
$$

and the continuity of $f$, it follows that $d(f z, S z)=0$, which implies that $f z \in S z$ since $S z$ is a closed subset of $X$ Similarly, we can prove that $g z \in T z$, that is, $z$ is a coincidence point of $g$ and $T$ This completes the proof

If we put $f=g=i_{X}$ ( the identity mapping on $X$ ) in Theorem 21 , we have the following

COROLLARY 2.2 [1] Let $(X, d)$ be a complete metric space and let $S, T: X \rightarrow C B(X)$ be $H$ continuous multi-valued mappings such that

$$
H^{p}(S x, T y) \leq \frac{c d(x, S x) d^{p}(y, T y)+b d(x, T y) d^{p}(y, S x)}{\delta(x, S x)+\delta(y, T y)}
$$

for all $x, y \in X$ for which $\delta(x, S x)+\delta(y, T y) \neq 0$, where $p \geq 1, b \geq 0$ and $1<c<2$. Then $S$ and $T$ have a common fixed point in $X$, that is, $z \in S z$ and $z \in T z$

Assuming that $f=g$ and $S=T$ on $X$ in Theorem 2.1, we have the following

COROLLARY 2.3. Let $(X, d)$ be a complete metric space and let $f: X \rightarrow X$ be a continuous single-valued mapping and $S: X \rightarrow C B(X)$ be an $H$-continuous multi-valued mapping such that

$S(X) \subset f(X)$,

$f$ and $S$ are continuous mappings,

$$
H^{p}(S x, T y) \leq \frac{c d(f x, S x) d^{p}(f y, S y)+b d(f x, S y) d^{p}(f y, S x)}{\delta(f x, S x)+\delta(f y, S y)}
$$

for all $x, y \in X$ for which $\delta(f x, S x)+\delta(f y, S y) \neq 0$, where $p \geq 1, b \geq 0$ and $1<c<2$ Then there exists a point $z \in X$ such that $f z \in S z$, i.e., $z$ is a coincidence point of $f$ and $S$

REMARK 2.1. If we put $p=1$ in Theorem 2.1, Corollaries 2.2 and 2.3 , we can obtain further corollaries.

\section{FIXED POINT THEOREMS FOR SINGLE-VALUED MAPPINGS}

In this section, using Theorem 2.1, we can obtain some fixed point theorems for single-valued mappings in a metric space

If $S$ and $T$ are single-valued mappings from a metric space $(X, d)$ into itself in Theorem 21 , we have the following

THEOREM 3.1. Let $(X, d)$ be a complete metric space Let $f, g, S$ and $T$ be continuous mappings from $X$ into itself such that

$$
S(X) \subset g(X) \text { and } T(X) \subset f(X),
$$

the pairs $f, S$ and $g, T$ are compatible mappings,

$$
\text { either (i) } \quad d^{p}(S x, T y) \leq \frac{c d(f x, S x) d^{p}(g y, T y)+b d(f x, T y) d^{p}(g y, S x)}{d(f x, S x)+d(g y, T y)}
$$

if $d(f x, S x)+d(g y, T y) \neq 0$ for all $x, y \in X$, where $p \geq 1, b \geq 0$ and $1<c<2$, or

$$
d(S x, T y)=0 \quad \text { if } \quad d(f x, S x)+d(g y, T y)=0 .
$$


Then $f, g, S$ and $T$ have a unique common fixed point $z$ in $X$ Further, $z$ is the unique common fixed point of $f, S$ and of $g, T$

PROOF. The existence of the point $w$ with $f w=S w$ and $g w=T w$ follows from Theorem 21 From (ii) of (3.3), since $d(f w, S w)+d(g w, T w)=0$, it follows that $d(S w, T w)=0$ and so $S w=f w=g w=T w$. By Lemma 12 , since $f$ and $S$ are compatible mappings and $f w=S w$, we have

$$
S f w=S S w=f S w=f f w,
$$

which implies that $d(f S w, S S w)+d(g w, T w)=0$ and, using the condition (ii) of (3.3), we have

$$
S f w=S S w=T w=g w=f w
$$

and so $f w=z$ is a fixed point of $S$. Further, (34) and (3 5) implies that

$$
S z=f S w=S S w=f z=z .
$$

Similarly, since $g$ and $T$ are compatible mappings, we have $T z=g z=z$. Using (ii) of ( 33 ), since $d(f z, S z)+d(g z, T z)=0$, it follows that $d(S z, T z)=0$ and so $S z=T z \quad$ Therefore, the point $z$ is a common fixed point of $f, g, S$ and $T$.

Next, we will show the uniqueness of the common fixed point $z$. Let $z^{\prime}$ be another common fixed point of $f$ and $S$. Using the condition (ii) of (3.3), since $d\left(f z^{\prime}, S z^{\prime}\right)+d(g z, T z)=0$, it follows that $d\left(z, z^{\prime}\right)=d\left(T z, S z^{\prime}\right)=0$ and so $z=z^{\prime}$. This completes the proof.

Now, we give an example of Theorem 3.1 with $p=1$ and $f=g$

EXAMPLE 3.1. Let $X=\{1,2,3,4\}$ be a finite set with the metric $d$ defined by

$$
\begin{aligned}
d(1,3)=d(1,4) & =d(2,3)=d(2,4)=1, \\
d(1,2) & =d(3,4)=2 .
\end{aligned}
$$

Define mappings $f, S, T: X \rightarrow X$ by

$$
\begin{gathered}
f(1)=1, f(2)=2, f(3)=4, f(4)=3 \\
S(1)=S(2)=S(4)=2, S(3)=3 \\
T(1)=T(2)=T(3)=T(4)=2 .
\end{gathered}
$$

From

$$
\begin{gathered}
S f(1)=S(1)=2=f(2)=f S(1), \\
S f(2)=S(2)=2=f(2)=f S(2) \\
d(S f(3), f S(3))=d(S(4), f(3))=d(2,4)<1<2=d(3,4)=d(S(3), f(3))
\end{gathered}
$$

and

$$
d(S f(4), f S(4))=d(S(3), f(2))=d(3,2)=1=d(2,3)=d(S(4), f(4)),
$$

it follows that $f$ and $S$ are weakly commuting mappings and so they are compatible. Clearly, $f, S$ and $T$ are continuous and

$$
S(x)=\{2,3\} \subset X=f(X), \quad T(X)=\{2\} \subset X=f(X) .
$$

Further, we can show that the inequality (i) of (3.3) holds with $c=\frac{3}{2}$ and $b=2$ and the condition (ii) of (3.3) holds only for the point 2. Therefore, all the conditions of Theorem 31 are satisfied and the point 2 is a unique common fixed point of $f, S$ and $T$.

REMARK 3.1. Theorem 3.1 assures that $f, g, S$ and $T$ have a unique common fixed point in $X$ However, either $f$ or $g$ or $S$ or $T$ can have other fixed points. Indeed, in Example $31, f$ and $S$ have two fixed points 
REMARK 3.2. From the proof of Theorem 31 , it follows that if the condition (ii) of $\left(\begin{array}{ll}3 & 3\end{array}\right)$ is omitted in the hypothesis of Theorem 3.1, then $f, g, S$ and $T$ have a coincidence point $w$, i e, $f w=g w=S w=T w$

If we put $f=g=i_{X}$ in Theorem 31 , we have the following.

COROLLARY 3.2. Let $(X, d)$ be a complete metric space and let $S, T: X \rightarrow X$ be continuous mappings such that

$$
\text { either (i) } \quad d^{p}(S x, T y) \leq \frac{c d(x, S x) d^{p}(y, T y)+b d(x, T y) d^{p}(y, S x)}{d(x, S x)+d(y, T y)}
$$

for all $x, y \in X$ if $d(x, S x)+d(y, T y) \neq 0$, where $p \geq 1, b \geq 0$ and $1<c<2$, or

$$
d(S x, T y)=0 \text { if } d(x, S x)+d(y, T y)=0 .
$$

Then $S$ and $T$ have a unique common fixed point $z$ in $X$

Assuming that $f=g$ and $S=T$ on $X$ in Theorem 3.1, we have the following.

COROLLARY 3.3. Let $(X, d)$ be a complete metric space and let $f, S: X \rightarrow X$ be continuous mappings such that

$S(X) \subset f(X)$,

$f$ and $S$ are compatible mappings,

$$
\text { either (i) } \quad d^{p}(S x, T y) \leq \frac{c d(x, S x) d^{p}(f y, S y)+b d(f x, S y) d^{p}(f y, S x)}{d(f x, S x)+d(y, S y)}
$$

for all $x, y \in X$ if $d(f x, S x)+d(f y, S y) \neq 0$, where $p \geq 1, b \geq 0$ and $1<c<2$, or

$$
d(S x, S y)=0 \quad \text { if } d(f x, S y)+d(f y, S y)=0 .
$$

Then $f$ and $S$ have a unique common fixed point $z$ in $X$

REMARK 3.3. (1) If $p=1$ in Corollary 3.2, we obtain the result of B Fisher [9].

(2) Theorem 3.1 is an extension of the results of M L. Diviccaro, S Sessa and B. Fisher [10]

REMARK 3.4. Conditions (3 6) and (3.7) are necessary in Corollary 3.3 (and so Theorem 3.1) [3]

EXAMPLE 3.1. Let $X=[0,1]$ with the Euclidean metric $d(x, y)=|x-y|$ and define two mappings $f, S: X \rightarrow X$ by

$$
S x=\frac{1}{4} \quad \text { and } \quad f x=\frac{1}{2} x
$$

for all $x \in X$. Note that $f$ and $S$ are continuous and $S(X)=\left\{\frac{1}{4}\right\} \subset\left[0, \frac{1}{2}\right]=f(X)$

Since $d(S x, S y)=0$ for all $x, y \in X$, all the conditions of Corollary 33 are satisfied except the compatibility of $f$ and $S$. In fact, let $\left\{x_{n}\right\}$ be a sequence in $X$ defined by $x_{n}=\frac{1}{2}$ for $n=1,2, \cdots$. Then we have

$$
\lim _{n \rightarrow \infty} f x_{n}=\lim _{n \rightarrow \infty} \frac{1}{2} x_{n}=\frac{1}{4}, \lim _{n \rightarrow \infty} S x_{n}=\lim _{n \rightarrow \infty} \frac{1}{4}=\frac{1}{4}
$$

but

$$
\lim _{n \rightarrow \infty} d\left(S f x_{n}, f S x_{n}\right)=\lim _{n \rightarrow \infty}\left|\frac{1}{4}-\frac{1}{8}\right|=\frac{1}{8} .
$$

Thus $f$ and $S$ are not compatible mappings But $f$ and $S$ have no common fixed points in $X$ 
ACKNOWLEDGMENT. The first author was supported in part by the Basic Science Research Institute Program, Ministry of Education, Korea, 1995, Project No. BSRI-95-1405

\section{REFERENCES}

[1] POPA, V., Theorems on multifunctions satisfying a rational inequality, Comment. Math. Untv. Carolinae, 24 (4) (1983), 673-680.

[2] SESSA, S., On a weak commutativity conditions of mappings in fixed point considerations, Publ. Inst. Math. (Beograd), 32 (46) (1982), 146-153

[3] JUNGCK, G, Compatible mappings and common fixed point, Internat. J. Math. Math. Scl. 9 (4) (1986), 771-779

[4] KANEKO, H , A Common fixed point of weakly commuting multi-valued mappings, Math. Japon., 33 (5) (1988), 741-744

[5] SINGH, S.L., HA, K.S and CHO, Y J., Coincidence and fixed points of nonlinear hybrid contractions, Internat. J. Math. Math. Scl., 12 (2) (1989), 247-256.

[6] KANEKO, H. and SESSA, S., Fixed point theorems for compatible multi-valued and single-valued mappings, Internat. J. Math. Math. Sci., 12 (1989), 257-262

[7] RUS, I.A., Fixed point theorems for multi-valued mappings in a complete metric space, Math. Japon., 20 (1975), 21-24

[8] NADLER, S.B , Multi-valued contraction mappings, Pacific J. Math., 20 (2) (1969), 475-488.

[9] FISHER, B., Theorems on mappings satisfying a rational inequality, Comment. Math. Univ. Carolinae, 19 (1978), 37-44.

[10] DIVICCARO, M.L., SESSA, S. and FISHER, B., Common fixed point theorems with a rational inequality, Bull. of Institute of Math., Academia Sinica, 14 (3) (1986), 277-285 


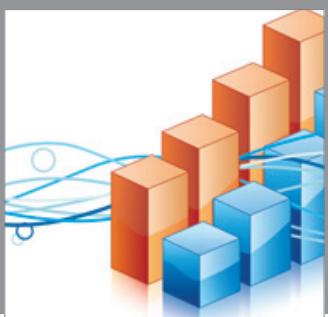

Advances in

Operations Research

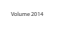

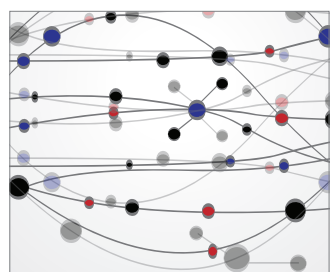

\section{The Scientific} World Journal
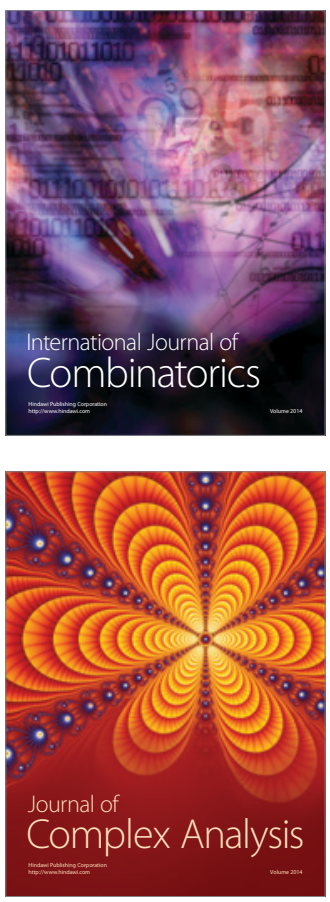

International Journal of

Mathematics and

Mathematical

Sciences
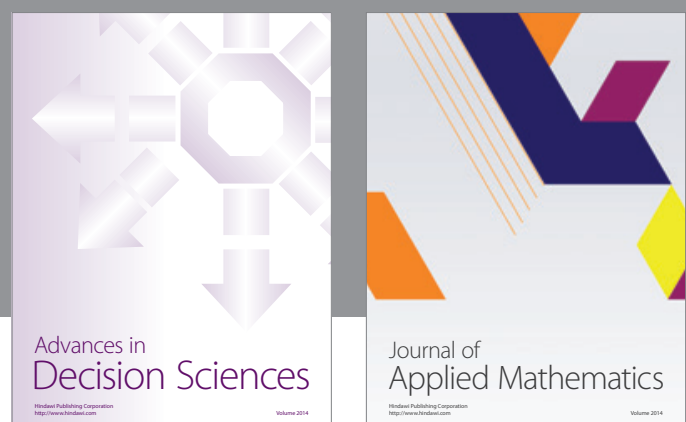

Journal of

Applied Mathematics
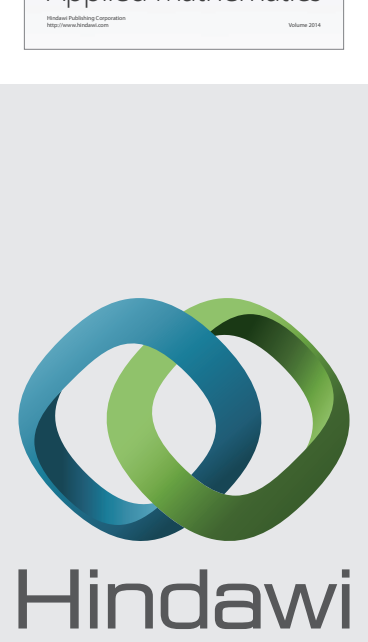

Submit your manuscripts at http://www.hindawi.com
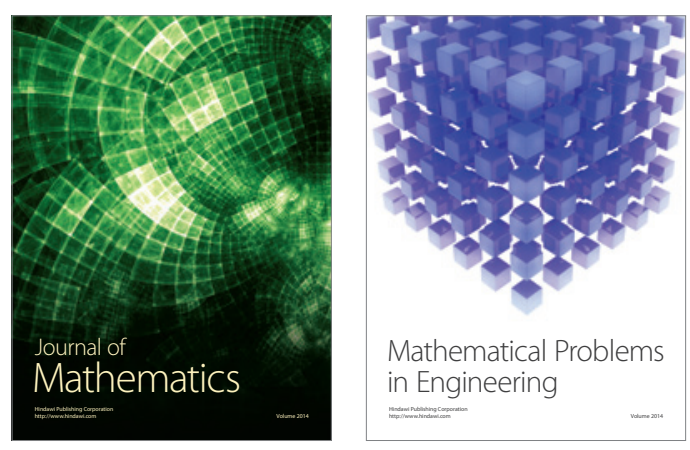

Mathematical Problems in Engineering
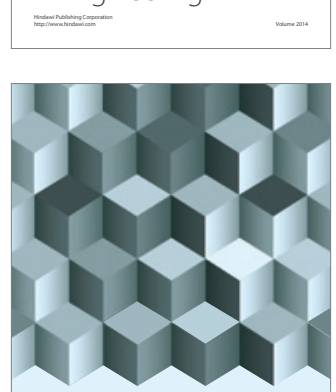

Journal of

Function Spaces
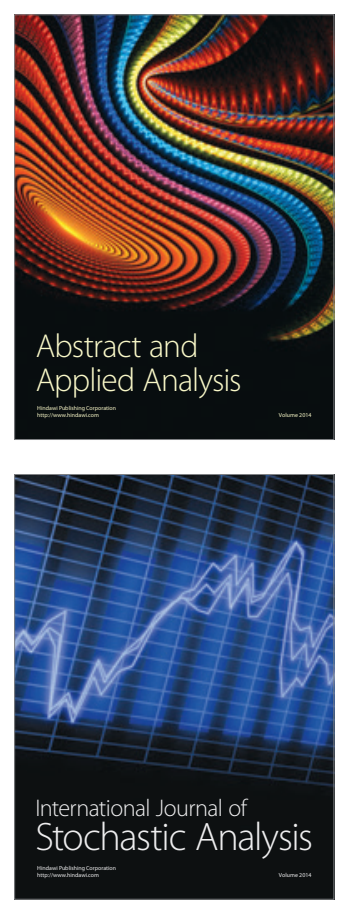

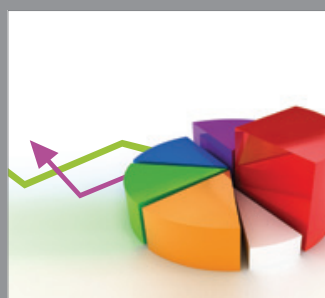

ournal of

Probability and Statistics

Promensencen
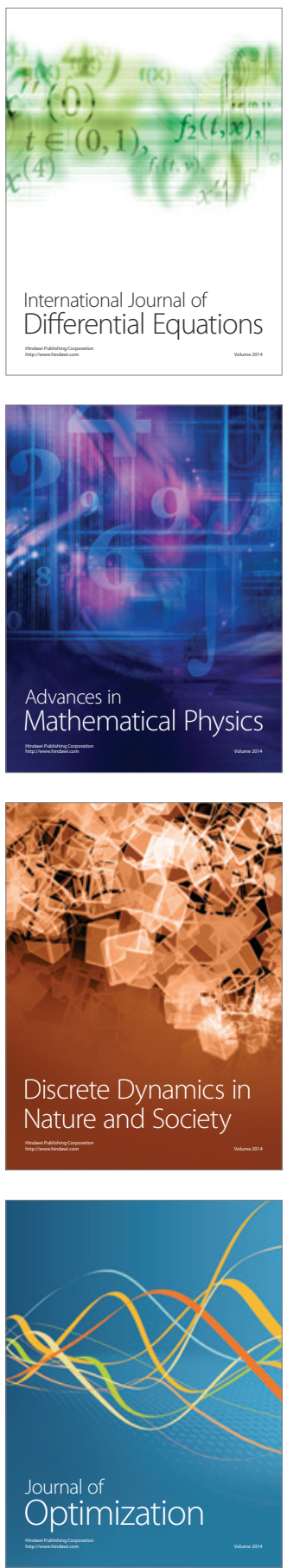